\title{
Electron-phonon interaction via Pekar mechanism in nanostructures
}

\author{
B. A. Glavin, V. A. Kochelap, and T. L. Linnik \\ V.E. Lashkarev Institute of Semiconductor Physics, \\ Ukrainian National Academy of Sciences, \\ Pr. Nauki 41, Kiev 03028, Ukraine \\ K. W. Kim \\ Department of Electrical and Computer Engineering, \\ North Carolina State University, Raleigh, NC 27695-7911, USA
}

\begin{abstract}
We consider an electron-acoustic phonon coupling mechanism associated with the dependence of crystal dielectric permittivity on the strain (the so-called Pekar mechanism) in nanostructures characterized by strong confining electric fields. The efficiency of Pekar coupling is a function of both the absolute value and the spatial distribution of the electric field. It is demonstrated that this mechanism exhibits a phonon wavevector dependence similar to that of piezoelectricity and must be taken into account for electron transport calculations in an extended field distribution. In particular, we analyze the role of Pekar coupling in energy relaxation in silicon inversion layers. Comparison with the recent experimental results is provided to illustrate its potential significance.
\end{abstract}

PACS numbers: 72.10.-d, 72.10.Di, 73.63.Hs 
The electron-phonon interaction is one of the fundamental problems in solid-state physics. For coupling with acoustic phonons in particular, much attention has been devoted to the two main mechanisms in semiconductors: the deformation potential and piezoelectric interaction. In the presence of an external electric field $E$, however, an additional process appears associated with the dependence of dielectric permittivity on the strain. Due to this dependence, an acoustic phonon can induce an effective ac electric field component and subsequently an additional coupling with electrons. This mechanism was initially introduced by Pekar [1, 2] for the problem of sound amplification by drifting electrons. It was shown later 3] that the Pekar mechanism of electron-phonon interaction is related directly to the electrostriction effect. The induced ac electric field has a piezo-like dependence on the phonon wavevector $q$ and can roughly be characterized by an effective piezo-constant

$$
\beta_{e f f} \sim \varepsilon^{2} E p
$$

where $p$ is the photoelasticity constant and $\varepsilon$ is the dielectric permittivity of the unstrained crystal. In ordinary bulk crystals with $E \lesssim 10^{4} \mathrm{~V} / \mathrm{cm}, \beta_{\text {eff }}$ is quite small. As a result, Pekar initially concentrated on the materials with a very large $\varepsilon$ (e.g., $\varepsilon \sim 2000$ for $\mathrm{BaTiO}_{3}$ ) and electrostriction effect. Efficient acousto-electric coupling for such materials was observed experimentally in the seventies [4].

As the dimension of the sample structure shrinks, interaction mechanisms that are not allowed in bulk materials can manifest themselves. For instance, modulation of the quantum well width and effective mass by strain gives rise to the so-called macroscopic deformation potential [5]. Similarly, the "traditional" mechanisms can exhibit different features. In this paper, we demonstrate that the Pekar mechanism is essential for nanostructures with strong confining electric fields [6, 7]. The interaction efficiency is dependent on the spatial scale of the electric field localization and the phonon wavelength. As a specific example, we consider low-temperature electron energy relaxation in n-type (100) Si inversion layers. Our calculation shows that due to the Pekar mechanism, the dissipated power in these nonpiezoelectric materials can exhibit a piezo-like $T^{3}$ dependence. This finding provides a clear explanation for a recent experimental observation of similar dependence [8].

Let us start with a general analysis of the Pekar mechanism for the case of a nonuniform electric field distribution. The variation of dielectric permittivity under strain $u_{i j}$ can be 
written in the form [9]:

$$
\delta \varepsilon_{i j}=\varepsilon_{i j}^{2} p_{i k l m} u_{l m}
$$

where $p_{i k l m}$ is the photoelasticity tensor (we could use the electrostriction tensor instead; however, the use of photoelasticity tensor allows one to extract explicitly the dependence of electrostriction on $\varepsilon$, which follows from the Clausius-Mossotti model [10]). The form of photoelasticity tensor is determined by the symmetry of the crystal. In the diamond and zinc-blende structures, there are only three independent nonzero components of this tensor denoted usually as $p_{11}, p_{12}$, and $p_{44}[9]$. Moreover, $\varepsilon_{i j}=\delta_{i j} \varepsilon$.

The electrostriction effect in a static external field $\boldsymbol{E}$ leads to appearance of an effective field $\tilde{\boldsymbol{E}}=-\nabla \tilde{\phi}$. The equation for potential $\tilde{\phi}$ can be derived from the equation for the electric displacement $\boldsymbol{D}$ :

$$
\nabla \cdot \boldsymbol{D}=0, D_{i}=\varepsilon_{i j} E_{k}^{(\Sigma)}
$$

where $\boldsymbol{E}^{(\Sigma)}=\boldsymbol{E}+\tilde{\boldsymbol{E}}$ is the total electric field in the crystal. Assuming that the strain is small, we obtain

$$
\nabla^{2} \tilde{\phi}=\frac{1}{\varepsilon} \frac{\partial}{\partial x_{i}}\left(\delta \varepsilon_{i k} E_{k}\right)
$$

In the simplest situation, the external electric field is $\boldsymbol{E}=(0,0, E(z))$, which corresponds to the confining field in a quantum well. We also assume an elastically uniform medium with the functional dependence of $u_{i j}, \delta \varepsilon_{i j} \sim \exp \left[i\left(-\omega t+q_{z} z+\boldsymbol{q}_{\|} \boldsymbol{\varrho}\right)\right]$ and $\tilde{\phi}=\tilde{\phi}(z) \exp \left[i\left(-\omega t+\boldsymbol{q}_{\|} \boldsymbol{\varrho}\right)\right]$, where $\boldsymbol{q}_{\|}=\left(q_{x}, q_{y}\right)$ and $\boldsymbol{\varrho}=(x, y)$ is the coordinate vector in the quantum well plane. Under these conditions, Eq. (4) simplifies as

$$
\frac{d^{2} \tilde{\phi}}{d z^{2}}-q_{\|}^{2} \tilde{\phi}=G(z), \quad G(z) \equiv\left(E(z) \frac{\partial \delta \varepsilon_{i z}}{\partial x_{i}}+\delta \varepsilon_{z z} \frac{d E(z)}{d z}\right) \frac{1}{\varepsilon},
$$

resulting in

$$
\tilde{\phi}=-\frac{1}{2 q_{\|}} e^{q_{\|} z} \int_{z}^{+\infty} G\left(z^{\prime}\right) e^{-q_{\|} z^{\prime}} d z^{\prime}-\frac{1}{2 q_{\|}} e^{-q_{\|} z} \int_{-\infty}^{z} G\left(z^{\prime}\right) e^{q_{\|} z^{\prime}} d z^{\prime} .
$$

It is important to note two important features of $\tilde{\phi}$. First, $\tilde{\phi}$ is not a plane wave as a function of $z$, which is a direct result of the assumed nonuniform character of the electric field. Second, $\tilde{\phi}$ strongly depends on the spatial domain of electric field localization, $d$. For $q_{z} d \gg 1$ and $q_{\|} d \gg 1$, the estimate of Eq. (10) applies. For $q_{z} d \ll 1$ and $q_{\|} d \ll 1$, however, the induced potential is suppressed substantially with a factor $q d$. 
Let us consider a specific example, namely, the process of energy relaxation in n-type (100) Si inversion layers at low temperatures. The built-in confining electric fields in such structures can be as high as several $M V / c m$. In this case, the role of Pekar mechanism is particularly important since silicon itself is not a piezoelectric material and the Pekar contribution is expected to be dominant at low temperatures, where the deformation potential interaction is less effective. Of course, the Pekar mechanism also provides a contribution to the momentum relaxation rate that determines the mobility. However, at low temperatures the momentum relaxes mainly due to the elastic scattering by various imperfections, and the contribution of phonons is hardly measurable. Thus, we concentrate on energy relaxation.

The electron potential well near the $\mathrm{Si} / \mathrm{SiO}_{2}$ interface and the electric field associated with the inversion layer are shown schematically in Fig. 1. The strong static electric field $E=-d \phi(z) / d z$ associated with the inversion layer confines the electrons in a thin silicon layer near the interface. The electrostatic potential $\phi(z)$, quantized electron levels and wavefunctions $\psi(\boldsymbol{r})=\chi(z) \exp (i \boldsymbol{k} \boldsymbol{\varrho})$ are determined from the self-consistent solution of Poisson and Schrödinger equations [6]. In the following, we assume that only the ground electron subband is populated at low temperatures.

The induced potential is determined by Eq. (5) . The boundary conditions at the $\mathrm{Si} / \mathrm{SiO}_{2}$ interface $(z=0)$ under a small strain are:

$$
\tilde{\phi}^{(s)}=\tilde{\phi}^{(i n s)}, \quad \varepsilon^{(s)} \tilde{E}_{z}^{(s)}+\delta \varepsilon_{z z}^{(s)} E^{(s)}=\varepsilon^{(i n s)} \tilde{E}_{z}^{(i n s)}+\delta \varepsilon_{z z}^{(i n s)} E^{(i n s)}
$$

where $\tilde{E}_{z}=-d \tilde{\phi} / d z$. For the considered geometry, the following photoelasticity terms are relevant:

$$
\begin{array}{r}
\delta \varepsilon_{x z}=2 \varepsilon^{2} p_{44} u_{x z}, \\
\delta \varepsilon_{y z}=2 \varepsilon^{2} p_{44} u_{y z}, \\
\delta \varepsilon_{z z}=\varepsilon^{2}\left(p_{11} u_{z z}+p_{12}\left(u_{y y}+u_{x x}\right)\right),
\end{array}
$$

where $x, y, z$ are the symmetry axis of the crystal. For simplicity, we disregard the mismatch of elastic and photoelasticity constants in $\mathrm{Si}$ and $\mathrm{SiO}_{2}$ layers. We also assume that the actual phonon wavelength exceeds the thickness of the depletion layer in silicon (see Fig. 1) and yet is much less than the thickness of the $\mathrm{SiO}_{2}$ layer. The former assumption can be justified at low enough temperatures, where the contribution of the Pekar mechanism exceeds that of the deformation potential. In case when the latter condition is violated, the induced 
potential becomes weak in accordance with the general analysis given above. In fact, this restricts the parameters of actual structures where the Pekar mechanism is important.

Under these assumptions, $\int d z \chi^{2}(z) \tilde{\phi}(z) \approx \tilde{\phi}(0)$, leading to

$$
\tilde{\phi}(0)=\frac{\varepsilon^{(s)} \varepsilon^{(i n s)}}{\varepsilon^{(s)}+\varepsilon^{(i n s)}} \frac{E_{s}}{q_{\|}+i q_{z}}\left(\left(p_{44} q_{\|}+i q_{z} p_{11}\right) u_{z}+\left(p_{44} \frac{q_{z}}{q_{\|}}+i p_{12}\right)\left(\boldsymbol{u}_{s} \boldsymbol{q}_{\|}\right)\right) .
$$

Here, $E_{s}$ and $\boldsymbol{u}_{s}$ are the electric field and the phonon displacement at the silicon side of the interface, respectively.

Using Eq. (9), we calculate the electron scattering rates $W_{k k^{\prime}}^{q_{z} \pm}$ for electron transition $\boldsymbol{k} \rightarrow \boldsymbol{k}^{\prime}$ with absorption (+) or emission (-) of an acoustic phonon:

$$
W_{\boldsymbol{k} k^{\prime}}^{q_{z} \pm}=\frac{\pi e^{2}\left(E_{s}\right)^{2}}{V \rho \omega_{q}}\left(\frac{\varepsilon^{(s)} \varepsilon^{(i n s)}}{\varepsilon^{(s)}+\varepsilon^{(i n s)}}\right)^{2}\left|\alpha_{l}(\theta)\right|^{2} \delta_{\boldsymbol{k}^{\prime}, \boldsymbol{k} \mp \boldsymbol{q}_{\|}} \delta\left(\epsilon_{k}-\epsilon_{k^{\prime}} \mp \hbar \omega_{q}\right)\left\{\begin{array}{l}
N_{q}+1 \\
N_{q}
\end{array}\right.
$$

where $\epsilon_{k}$ is the electron energy and $N_{q}$ represents the Planck population of the phonon modes characterized by the lattice temperature $T$. The index $l$ of the function $\left|\alpha_{l}(\theta)\right|$ denotes the type of the phonon modes. In particular,

$$
\begin{gathered}
\left|\alpha_{L A}(\theta)\right|^{2}=\left(p_{11}^{2}+\left(4 p_{44}^{2}-2 p_{11}^{2}+2 p_{11} p_{12}\right) \sin ^{2} \theta+\left(p_{11}^{2}+p_{12}^{2}-2 p_{11} p_{12}-4 p_{44}^{2}\right) \sin ^{4} \theta\right), \\
\left|\alpha_{T A}(\theta)\right|^{2}=\left(p_{44}^{2}\left(\sin ^{2} \theta-\cos ^{2} \theta\right)^{2}+\left(p_{11}-p_{12}\right)^{2} \sin ^{2} \theta \cos ^{2} \theta\right),
\end{gathered}
$$

where $\theta$ is the angle between the phonon wave vector $\boldsymbol{q}$ and the $z$ axis, $V$ and $\rho$ are the normalizing volume and density, respectively, and $\omega_{q}=s_{l, t} q$ is the phonon frequency. $\alpha_{T A}$ corresponds to the contribution of the transverse phonons with a vertical polarization ( $\boldsymbol{u}$ lies in the plane formed by the $z$ axis and $\boldsymbol{q}$ ); the contribution of the phonons with a horizontal polarization ( $\boldsymbol{u}$ is parallel to the interface) is zero. The power $Q$ dissipated by electrons is given as [1]:

$$
Q=g S^{-1} \Sigma_{\boldsymbol{k}, k^{\prime}, q_{z}}\left(\epsilon_{k}-\epsilon_{k^{\prime}}\right)\left(W_{\boldsymbol{k} k^{\prime}}^{q_{z} ;+}+W_{\boldsymbol{k} k^{\prime}}^{\left(q_{z} ;-\right)}\right) f_{T_{e}}(\boldsymbol{k})\left(1-f_{T_{e}}\left(\boldsymbol{k}^{\prime}\right)\right),
$$

where $g$ is the electron degeneracy $(g=4$ in a (100) inversion layer taking into account both the spin and valley degeneracy) and $S$ is the cross sectional area for normalization. In the expression for $Q$, we assume that the electron-electron interaction establishes a Fermi distribution for electrons with an electron temperature $T_{e}$.

At a low temperature, the small-angle (Bloch-Grünizen) scattering regime is realized. For degenerate electrons after the standard transformations, we obtain a piezo-like temperature 
dependence:

$$
\begin{array}{r}
Q=C\left(T_{e}^{3}-T^{3}\right), \\
C=\left(C_{L A}+C_{T A}\right) \frac{1}{\pi^{7 / 2}} \frac{m^{2}\left(E_{s}\right)^{2}}{\hbar^{5} n_{e}^{1 / 2}} \frac{e^{2}}{\rho} \zeta(3)\left(\frac{\varepsilon^{(s)} \varepsilon^{(i n s)}}{\varepsilon^{(s)}+\varepsilon^{(i n s)}}\right)^{2}, \\
C_{L A}=\frac{1}{s_{l}^{2}} \frac{\pi}{8}\left(4 p_{44}^{2}+2 p_{11} p_{12}+3 p_{11}^{2}+3 p_{12}^{2}\right), \\
C_{T A}=\frac{1}{s_{t}^{2}} \frac{\pi}{8}\left(4 p_{44}^{2}+\left(p_{11}-p_{12}\right)^{2}\right),
\end{array}
$$

where $m$ is the electron effective mass for the in-plane motion, $n_{e}$ is the electron concentration in the inversion layer, and $\zeta$ is the Riemann zeta-function, $\zeta(3) \approx 1.20$.

For a numerical estimation, we use the following material parameters: $\varepsilon_{S i}=12, \rho=$ $2 \mathrm{~g} / \mathrm{cm}^{3}, \varepsilon_{\mathrm{SiO}_{2}}=4, s_{l}=9 \times 10^{5} \mathrm{~cm} / \mathrm{s}, s_{t}=5.4 \times 10^{5} \mathrm{~cm} / \mathrm{s}$, and $m=0.19 m_{0}$ [for the (100) interface]. The values of the photoelasticity tensor are $p_{11}=-0.093, p_{12}=0.026$, and $p_{44}=-0.05[12]$. As mentioned previously, the electron density and electric field at the interface must be determined by a self-consistent procedure. As a rough estimate, we adopt $E_{s}=3 \times 10^{5} \mathrm{~V} / \mathrm{cm}$ and $n_{e}=5 \times 10^{11} \mathrm{~cm}^{-2}$, which provide $C=10^{-4} \mathrm{~W} / \mathrm{K}^{3} \mathrm{~m}^{2}$. This corresponds to the energy relaxation time of about $1 \mathrm{~ms}$ at the electron temperature of $T_{e} \sim 1 \mathrm{~K}$ and $T \sim T_{e}$.

In a recent experiment on silicon metal-oxide-semiconductor field-effect transistors [8], the cubic dependence of $Q$ on $T_{e}$ was observed at low temperatures, which was followed by a $T_{e}^{5}$ dependence for $T_{e}>0.6 \mathrm{~K}$ characteristic for the deformation potential coupling. The authors of Ref. 8 attributed the $T_{e}^{3}$ dependence to the appearance of effective piezoelectric properties due to the specific structure of the interface. Indeed, the interface reduces the symmetry of the system which can give rise to nonzero piezoelectricity in the vicinity of interface. However, for the phonon wavelength exceeding the thickness of this "piezoelectric" layer, the piezoelectric potential induced by the phonon is suppressed (this is similar to the case of the Pekar potential for narrow regions of electric field localization). Therefore, the observed cubic dependence is more likely to be due to the Pekar mechanism. Note also, that the obtained above value of $C$ is close to that measured experimentally [8].

In summary, we show that the Pekar mechanism of electron-phonon interaction (related to the electrostriction effect) can become important in nanostructures due to the presence of strong confining electric fields and must be considered along with the deformation potential and piezoelectric mechanisms. The effectiveness of Pekar coupling depends on both the 
FIG. 1: (a) Schematic illustration of the potential well in a n-type Si inversion layer. The thickness of the depletion layer is denoted by $d_{d e p l}$. (b) $z$-dependence of the confining electric field in the inversion layer.

absolute value and the spatial distribution of the electric field. An estimation of power dissipation by this mechanism in a silicon inversion layer is in good agreement with a recent experiment.

We are indebted to V.N. Piskovoi for many valuable comments and discussions. The work was supported in part by AFOSR and CRDF (grant UE2-2439-KV-02).

[1] S. I. Pekar, Zh. Eksp. Teor. Fiz. 49, 621 (1965) [Sov. Phys. JETP 22, 431 (1966)].

[2] A. A. Demidenko, V. N. Piskovoi, S. I. Pekar, and B. E. Tsekvava, Zh. Eksp. Teor. Fiz. 50, 124 (1966) [Sov. Phys. JETP 23, 84 (1966)].

[3] Y.V. Gulyaev, Fiz. Tverdogo Tela 9, 1816 (1967) [Sov. Phys. Solid State 9, 1425 (1967)].

[4] N.K. Zhabitenko, I.Ya. Kucherov, E.G. Miselyuk, S.I. Pekar, and N.S Chernaya, Pis'ma Zh. Eksp. Teor. Fiz. 14, 458 (1971) [Sov. Phys. JETP Lett. 14, 312 (1971)].

[5] F.T. Vasko and V.V. Mitin, Phys. Rev. B 52, 1500 (1995); P.A. Knipp and T.L. Reinecke, Phys. Rev. B 52, 5923 (1995); V.I. Pipa, V.V. Mitin, and M.A. Stroscio, Appl. Phys. Lett. 74, 1585 (1999).

[6] T. Ando, A. B. Fowler, and F. Stern, Rev. Mod. Phys. 54, 437 (1982).

[7] O. Ambacher, J. Majewski, C. Miskys, A. Link, M. Hermann, M. Eickhoff, M. Stutzmann, F. Bernardini, V. Fiorentini, V. Tilak, B. Schaff, and L.F. Eastman, J. Phys.: Condens. Matter 14, 3399 (2002).

[8] O. Prus, M. Reznikov, U. Sivan, and V. Pudalov, Phys. Rev. Lett. 88, 016801 (2002).

[9] J.W. Tucker and V.W. Rampton, Microwave Ultrasonics in Solid State Physics (NorthHolland, Amsterdam, 1972).

[10] N.W. Ashkroft and N.D. Mermin, Solid State Physics (Saunders College, Philadelphia, 1976).

[11] V.E. Gantmakher and Y.B. Levinson, Carrier Scattering in Metals and Semiconductors (North Holland, Amsterdam, 1987).

[12] Z.H. Levine, H. Zhong, S. Wei, D.C. Allan, and J.W. Wilkins, Phys. Rev. B 45, 4131 (1992). 

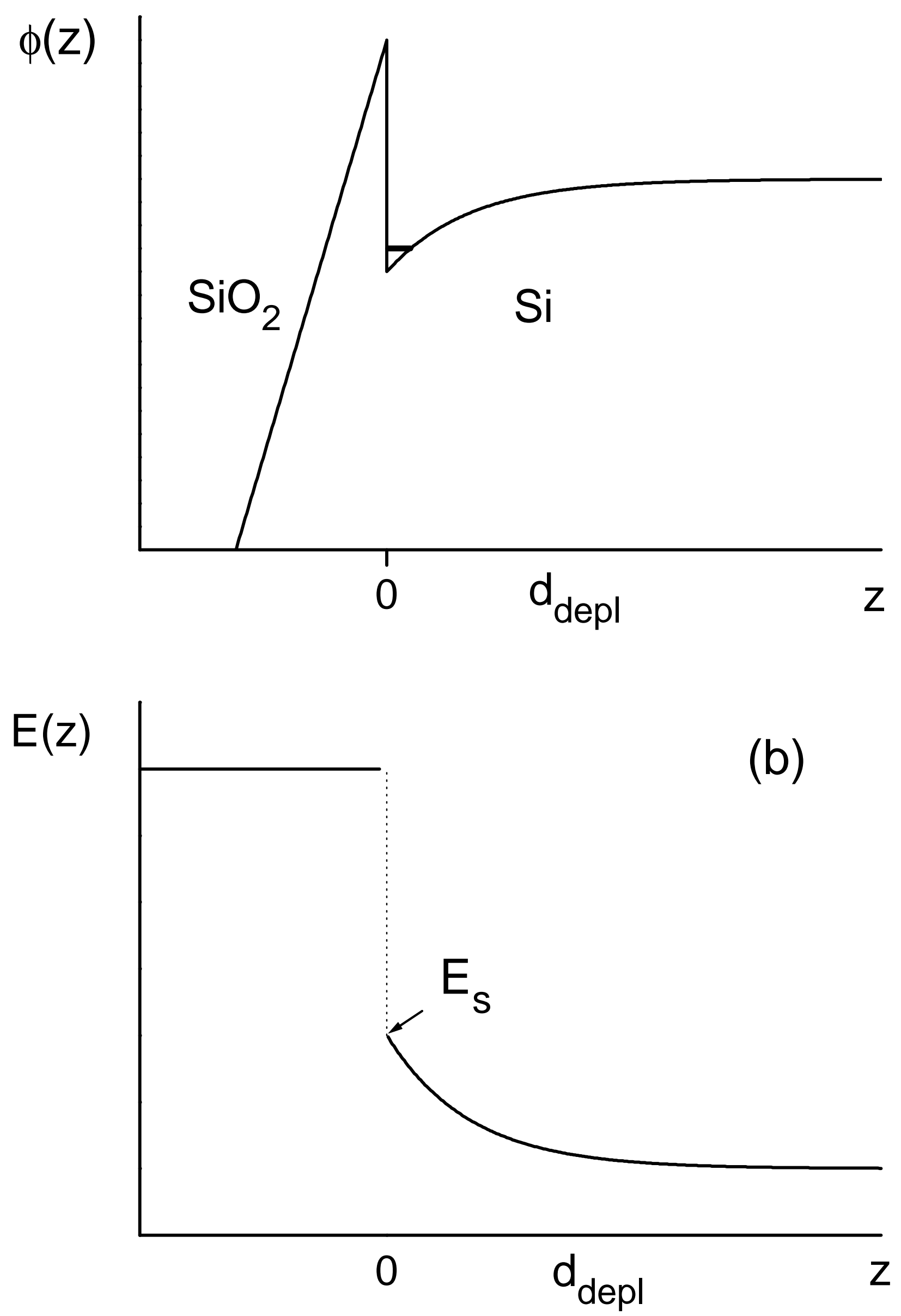